\title{
Phonons and Electron-Phonon Scattering in Carbon Nanotubes
}

\author{
Hidekatsu SuZuURA and Tsuneya ANDO \\ Institute for Solid State Physics, University of Tokyo \\ 5-1-5 Kashiwanoha, Kashiwa, Chiba 277-8581, Japan
}

\begin{abstract}
Electron-phonon scattering is studied within an effective-mass theory. A continuum model for acoustic phonons is introduced and electron-phonon interaction due to modification of band structure is derived as well as a normal deformation potential. In a metallic nanotube, the deformation potential does not participate in electron scattering and a metallic nanotube becomes nearly a one-dimensional ballistic conductor even at room temperature. A resistivity determined by small band-structure interaction depends on the chirality at low temperatures. A magnetic field perpendicular to the axis induces electron scattering by the deformation potential, giving rise to huge positive magnetoresistance.
\end{abstract}

\section{INTRODUCTION}

Carbon nanotubes (CN's) are quasi-one-dimensional materials made of $s p^{2}$-hybridized carbon networks. ${ }^{1}$ Electronic structure of a single $\mathrm{CN}$ has been studied theoretically, which predicted that $\mathrm{CN}$ becomes either metallic or semiconducting depending on its chiral vector, i.e., boundary conditions in the circumference direction. ${ }^{2-11}$ These predictions have been confirmed by Raman experiments $^{12}$ and direct measurements of local density of states by scanning tunneling spectroscopy. ${ }^{13-15}$ Transport properties are particularly interesting because of their unique topological structures. ${ }^{16}$ In this paper we focus on electron-phonon scattering as the main origin of resistivity and reveal its chirality and magnetic-field dependence.

For impurity scattering, it was shown theoretically that there is no backscattering for impurity potentials with a range larger than the lattice spacing in metallic CN's. ${ }^{17}$ This intriguing fact was related to Berry's phase acquired by a rotation in the wave-vector space in the system described by a $\boldsymbol{k} \cdot \boldsymbol{p}$ Hamiltonian. ${ }^{18}$ The absence of backward scattering has been confirmed by numerical calculations in a tight-binding model. ${ }^{19}$ There have been some reports on experiments which seem to support this theoretical prediction. ${ }^{20,21}$

Effects of scattering by a lattice vacancy in armchair nanotubes have been studied within a tight-binding model. ${ }^{22,23}$ It has been shown that the conductance at $\varepsilon=0$ in the absence of a magnetic field is quantized into zero, one, or two times of the conductance quantum $e^{2} / \pi \hbar$ for a vacancy consisting of three $\mathrm{B}$ carbon atoms around an A atom, of a single $\mathrm{A}$ atom, and of a pair of A and B atoms, respectively. ${ }^{23}$ Numerical calculations were performed for about $1.5 \times 10^{5}$ different kinds of vacancies and demonstrated that such quantization is quite general. ${ }^{24}$ This rule was analytically derived in a $\boldsymbol{k} \cdot \boldsymbol{p}$ scheme later. ${ }^{25,26}$

Phonon scattering is another main origin of the resistivity and gives dominant contributions usually at high temperature. Phonons contributing to the electron scattering and resistivity are those of long wavelengths which can be described well by continuum models. Although electronic properties have been understood by those of the graphite plane using a periodic boundary condition, the phonon modes of nanotubes are not simply given by the zone-folded modes of planes because they fail to give breathing modes. ${ }^{6}$ In this paper we shall construct a continuum model suitable for a correct description of long-wavelength acoustic phonons.

A lattice deformation gives rise to a diagonal energy shift called deformation potential in a matrix $\boldsymbol{k} \cdot \boldsymbol{p}$ Hamiltonian. In addition to the deformation potential it causes small off-diagonal terms arising from a modification in the local band structure due to change in the bond-length between neighboring carbon atoms. In the absence of a magnetic field, the former deformation potential gives no backscattering and the resistivity is determined by the latter band-structure term. A magnetic field changes the situation drastically and leads to an appreciable amount of backscattering due to the deformation potential. This leads to increase of the resistivity in a magnetic field, or positive magnetoresistance, in metallic CN's.

In this paper, we shall calculate the phonon-limited resistance of metallic CN's and demonstrate a huge positive magnetoresistance. The case of semiconducting C$\mathrm{N}$ 's will be touched upon very briefly. In $\S 2$ a continuum model for long-wavelength phonons is introduced and resulting modes are compared with those of microscopic calculations. In $\S 3$ an effective Hamiltonian for electronphonon interaction is derived in a $\boldsymbol{k} \cdot \boldsymbol{p}$ or effective-mass approximation. In $\S 4$ the resistivity is calculated and in $\S 5$ the magnetoresistance is calculated. The continuum phonon model is derived from a microscopic valenceforce-field model in $\S 6$. A discussion and short summary are given in $\S \S 7$ and 8 , respectively. Very preliminary account of a part of the results has been published. ${ }^{27,28}$

\section{LONG-WAVELENGTH PHONON}

As shown in Fig. 1, we choose the $x$ axis in the circumference direction, $y$ in the axis direction, and $z$ in the direction normal to the cylinder surface. The radius of the nanotube is denoted as $R$ and the circumference $L$, i.e., $R=L / 2 \pi$. Acoustic phonons important in the electron scattering are known to be described well by a continuum model. Two-dimensional graphites show isotropic elasticity and the potential-energy functional for in-plane displacement $\boldsymbol{u}(\boldsymbol{r})=\left(u_{x}, u_{y}\right)$ is written as

$U[\boldsymbol{u}]=\int d x d y \frac{1}{2}\left(B\left(u_{x x}+u_{y y}\right)^{2}+\mu\left[\left(u_{x x}-u_{y y}\right)^{2}+4 u_{x y}^{2}\right]\right)$,

with strain tensors

$$
u_{x x}=\frac{\partial u_{x}}{\partial x}, u_{y y}=\frac{\partial u_{y}}{\partial y}, 2 u_{x y}=\frac{\partial u_{x}}{\partial y}+\frac{\partial u_{y}}{\partial x} .
$$

The parameters $B=\lambda+\mu$ and $\mu$ denote the bulk modulus 
and the shear modulus for a graphite sheet $(\lambda$ and $\mu$ are Lamé constants).

It should be noted that the displacement $\boldsymbol{u}(\boldsymbol{r})$ is not identical to that of the atom located at $\boldsymbol{r}$ and this is important for quantitative evaluation of electron-phonon coupling although it has not been paid much attention to. This phenomenological model can be derived also from a simple valence-force-field model as shown in $\S 6$.

For CN's, in-plane and out-of-plane modes are no longer separate and the normal component of displacement should be considered on an equal footing. According to the theory of elasticity in the cylindrical coordinate system, ${ }^{29}$ the nonzero curvature of the nanotube leads to

$$
u_{x x}=\frac{\partial u_{x}}{\partial x}+\frac{u_{z}}{R}
$$

where $u_{z}$ is the displacement perpendicular to the cylinder surface. With this correction the potential-energy functional (2.1) has full continuous symmetry for cylindrical geometry and its absence breaks invariance to the uniform translation in a direction normal to the tube axis as will be further discussed below.

The effect of a finite curvature of the nanotube can be derived also from a microscopic valence-force-field model. What is the key point is to regard $R^{-1}$ as a small parameter in perturbative expansions as well as wavenumber. Long but straight-forward calculations reveal that the curvature effect generates the above expression of $u_{x x}$ within the second-order perturbation in terms of wavenumber and $R^{-1}$.

The corresponding kinetic energy is written as

$$
K[\boldsymbol{u}]=\int d x d y \frac{M}{2}\left[\left(\dot{u}_{x}\right)^{2}+\left(\dot{u}_{y}\right)^{2}+\left(\dot{u}_{z}\right)^{2}\right],
$$

where $M$ is the mass density given by the carbon mass per unit area, $M=3.80 \times 10^{-7} \mathrm{~kg} / \mathrm{m}^{2}$. The corresponding equations of motion are given by

$$
\begin{aligned}
& M \ddot{u}_{x}=(B+\mu) \frac{\partial^{2} u_{x}}{\partial x^{2}}+\mu \frac{\partial^{2} u_{x}}{\partial y^{2}}+B \frac{\partial^{2} u_{y}}{\partial x \partial y}+\frac{B+\mu}{R} \frac{\partial u_{z}}{\partial x} \\
& M \ddot{u}_{y}=B \frac{\partial^{2} u_{x}}{\partial x \partial y}+(B+\mu) \frac{\partial^{2} u_{y}}{\partial y^{2}}+\mu \frac{\partial^{2} u_{y}}{\partial x^{2}}+\frac{B-\mu}{R} \frac{\partial u_{z}}{\partial y} \\
& M \ddot{u}_{z}=-\frac{B+\mu}{R} \frac{\partial u_{x}}{\partial x}-\frac{B-\mu}{R} \frac{\partial u_{y}}{\partial y}-\frac{B+\mu}{R^{2}} u_{z} .
\end{aligned}
$$

The phonon modes are specified by the wave vector along the circumference $\chi(n)=2 \pi n / L$ and that along the axis $q$ as

$$
\boldsymbol{u}(\boldsymbol{r})=\boldsymbol{u}_{n q} \exp [i \chi(n) x+i q y] .
$$

When $n=0$ and $\chi=0$, in particular, the eigen equation becomes

$M \omega^{2}\left(\begin{array}{l}u_{x} \\ u_{y} \\ u_{z}\end{array}\right)=\left(\begin{array}{ccc}\mu q^{2} & 0 & 0 \\ 0 & (B+\mu) q^{2} & -i(B-\mu) q R^{-1} \\ 0 & i(B-\mu) q R^{-1} & (B+\mu) R^{-2}\end{array}\right)\left(\begin{array}{l}u_{x} \\ u_{y} \\ u_{z}\end{array}\right)$

which has three eigenmodes called twisting, stretching, and breathing.

The twisting mode $\left(u_{x} \neq 0, u_{y}=u_{z}=0\right)$ is made of pure circumference-directional deformation and its velocity $v_{T}$ is equal to that of the TA mode of a graphite sheet,

$$
\omega_{T}(q)=v_{T} q, \quad v_{T}=v_{T}^{G}=\sqrt{\frac{\mu}{M}} .
$$

In the long wavelength limit $q=0$, the radial deformation $u_{z}$ generates a breathing mode with a frequency

$$
\omega_{B}=\sqrt{\frac{B+\mu}{M}} \frac{1}{R}
$$

which is inversely proportional to the radius $R$. In the case $|q R| \ll 1$, the deformation in the nanotube-axis direction generates stretching modes. When $\omega \ll \omega_{B}$, we have from the last equation of eq. (2.7)

$$
u_{z} \approx-i \frac{B-\mu}{B+\mu} q R u_{y}
$$

Upon substitution of this into the second equation of eq. (2.7) for $u_{y}$, we have

$$
\omega_{S}=v_{S} q, \quad v_{S}=\sqrt{\frac{4 B \mu}{(B+\mu) M}} .
$$

The velocity $v_{S}$ is usually smaller than that of the LA mode of the graphite $v_{L}^{G}=\sqrt{(B+\mu) / M}$. We set $v_{L}^{G}=$ $21.0 \mathrm{~km} / \mathrm{s}$ and $v_{T}^{G}=12.3 \mathrm{~km} / \mathrm{s}$, and we obtain $v_{S}=19.9$ $\mathrm{km} / \mathrm{s}, v_{T}=12.3 \mathrm{~km} / \mathrm{s}$, and $\hbar \omega_{B}=2.04 \times 10^{-2} \mathrm{eV}$, or $237 \mathrm{~K}$ for the so-called $(10,10)$ armchair CN with $R=6.79 \AA$.

The above model is too simple when dealing with modes with $n \neq 0$. In order to see this fact explicitly, we shall consider the case with $q=0$. In this case there is a displacement given by

$$
\begin{aligned}
& u_{x}=-\frac{u}{n} \sin \frac{2 \pi n x}{L}=-\frac{u}{n} \sin \frac{n x}{R}, \\
& u_{z}=u \cos \frac{2 \pi n x}{L}=u \cos \frac{n x}{R},
\end{aligned}
$$

with arbitrary $u$. This displacement gives $u_{x x}=0$ identically and also $u_{y y}=u_{x y}=0$, giving rise to the vanishing frequency. For $n= \pm 1$, this vanishing frequency is absolutely necessary because the displacement corresponds to a uniform shift of a nanotube in a direction perpendicular to the axis. For $|n|>1$, on the other hand, the displacement corresponds to a deformation of the cross section of the nanotube as shown in Fig. 2. Such deformations should have nonzero frequency in actual graphite because otherwise CN cannot maintain a cylindrical form.

Actually, we have to consider the potential energy due to curvature change of the nanotube surface. Here, we introduce a phenomenological potential-energy functional of $\boldsymbol{u}(\boldsymbol{r})$. There are two types of curvature characterizing deviations from flatness of a surface: mean curvature and Gaussian curvature. ${ }^{30}$ The integration of the Gaussian curvature over entire surface gives a topological invariant number. Therefore, change of the mean curvature is a unique candidate for the effective potential as long as the topology of the nanotube remains fixed 
under lattice deformation.

The mean curvature $H_{c}[\boldsymbol{u}]$ of a nanotube under deformation $\boldsymbol{u}$ is given by

$$
2 H_{c}[\boldsymbol{u}]=\frac{1}{R}-\left(\frac{\partial^{2}}{\partial x^{2}}+\frac{1}{R^{2}}+\frac{\partial^{2}}{\partial y^{2}}\right) u_{z}
$$

up to the first-order term of displacement, where $R^{-1}$ is the mean curvature in the absence of displacement. We adopt the square of its deviation from the equilibrium value as a potential and it is written as

$$
U_{c}[\boldsymbol{u}]=\frac{1}{2} a^{2} \Xi \int d x d y\left[\left(\frac{\partial^{2}}{\partial x^{2}}+\frac{1}{R^{2}}+\frac{\partial^{2}}{\partial y^{2}}\right) u_{z}\right]^{2},
$$

where $\Xi$ is a force constant for curvature deviation. For $2 \mathrm{D}$ graphites $(R \rightarrow \infty)$, this correctly reproduces the dispersion relation of out-of-plane modes. As for nanotubes, the presence of the term $R^{-2}$ guarantees that the deformation with $n= \pm 1$ given in eq. (2.12) has a vanishing frequency.

This curvature energy is of the order of the fourth power of the wave vector and therefore is much smaller than $U[\boldsymbol{u}]$ as long as $q R \ll 1$ for $n=0$ and \pm 1 but becomes appreciable for $|n|>1$. After all, three parameters $B, \mu$, and $\Xi$ are necessary for describing long-wavelength acoustic phonons in CN's and, in this paper, those of a bulk graphite determined by its phonon dispersion relations are put into use as given in $\S 6$.

Figure 3 shows phonon dispersions of a $(10,10)$ armchair $\mathrm{CN}$ calculated in this continuum model. The solid lines show the modes with $n=0$, i.e., the twist mode with a linear dispersion, and the stretch and breathing modes coupled with each other when crossing. When we ignore curvature effects as shown in Fig. 3(a), there are modes with frequency $\propto q^{2}$ for all nonzero $n$. When curvature effects are taken into account as shown in Fig. 3(b), on the other hand, the frequency of these modes becomes nonzero at $q=0$ except for the mode with $n= \pm 1$.

This mode with $n= \pm 1$ corresponds to bending motion of a cylinder and should have a $q^{2}$ dispersion. The modes with $n=0$ are essentially not affected by curvature effects except when $q R \gg 1$. The results with curvature effects are in good agreement with the results of microscopic calculations. ${ }^{31}$ The result for the bending mode given in ref. 31 looks almost linear as a function of $q$ and are likely to be caused by an inappropriate choice of force-constant values.

\section{ELECTRON-PHONON INTERACTION}

\section{A. Deformation Potential}

A long-wavelength acoustic phonon gives rise to an effective potential called the deformation potential

$$
V_{1}=g_{1}\left(u_{x x}+u_{y y}\right)
$$

proportional to a local dilation or dilatation $\Delta(\boldsymbol{r})=u_{x x}+$ $u_{y y}$. This term appears as a diagonal term in the matrix Hamiltonian in the effective-mass approximation. The coupling constant $g_{1}$ is called the deformation potential and its very rough value can be estimated in a nearly-free electron model.

Consider a rectangular area $a \times a$. In the presence of a lattice deformation, the area $S$ changes into $S+$ $\delta S(\boldsymbol{r})$ with $\delta S(\boldsymbol{r})=a^{2} \Delta(\boldsymbol{r})$. Therefore, the ion density changes locally by $n_{0} \rightarrow n_{0}[1-\Delta(\boldsymbol{r})]$. The electron density should change in the same manner due to the charge neutrality condition. Consider a two-dimensional electron gas. The potential energy $\delta \varepsilon(\boldsymbol{r})$ corresponding to the density change should satisfy $\delta \varepsilon(\boldsymbol{r}) D\left(\varepsilon_{F}\right)=n_{0} \Delta(\boldsymbol{r})$, where $D\left(\varepsilon_{F}\right)$ is the density of states at the Fermi level $D(\varepsilon)=m / \pi \hbar^{2}$ independent of energy, where $m$ is the free-electron mass. Therefore, $n_{0}=D\left(\varepsilon_{F}\right) \varepsilon_{F}$, leading to

$$
\delta \varepsilon(\boldsymbol{r})=\varepsilon_{F} \Delta(\boldsymbol{r}) .
$$

This shows $g_{1}=\varepsilon_{F}$ in contrast to $g_{1}=(2 / 3) \varepsilon_{F}$ in three dimension. In the two-dimensional graphite, the electron gas model may not be so appropriate but can be used for a very rough estimation of $g_{1}$ as the Fermi energy measured from the bottom of the valence bands ( $\sigma$ bands), i.e., $20-30 \mathrm{eV}$. The deformation potential $g_{1}$ in the bulk graphite has been known to be about $16 \mathrm{eV} .^{32}$

\section{B. Bond-Length Change}

A tight-binding equation of motion is given by

$$
\begin{aligned}
& \varepsilon \psi_{A}\left(\boldsymbol{R}_{A}\right)=-\sum_{l} \gamma_{\boldsymbol{R}_{A}, \boldsymbol{R}_{A}-\vec{\tau}_{l}} \psi_{B}\left(\boldsymbol{R}_{A}-\vec{\tau}_{l}\right), \\
& \varepsilon \psi_{B}\left(\boldsymbol{R}_{B}\right)=-\sum_{l} \gamma_{\boldsymbol{R}_{B}, \boldsymbol{R}_{B}+\vec{\tau}_{l}} \psi_{A}\left(\boldsymbol{R}_{B}+\vec{\tau}_{l}\right),
\end{aligned}
$$

where the energy origin has been chosen at the energy level of the $p_{z}$ orbital, $\psi_{A}$ and $\psi_{B}$ are the amplitude at a carbon $\mathrm{A}$ site $\boldsymbol{R}_{A}$ and a $\mathrm{B}$ site $\boldsymbol{R}_{B}$, respectively, $\gamma_{\boldsymbol{R}_{A}, \boldsymbol{R}_{A}-\vec{\tau}_{l}}$ and $\gamma_{\boldsymbol{R}_{B}, \boldsymbol{R}_{B}+\vec{\tau}_{l}}$ are the transfer integral between neighboring atoms, $\vec{\tau}_{1}=(0,1 / \sqrt{3}) a, \vec{\tau}_{2}=$ $(-1 / 2,1 / 2 \sqrt{3}) a$, and $\vec{\tau}_{3}=(1 / 2,-1 / 2 \sqrt{3}) a$. Let $\boldsymbol{u}_{A}\left(\boldsymbol{R}_{A}\right)$ and $\boldsymbol{u}_{B}\left(\boldsymbol{R}_{B}\right)$ be a lattice displacement at A and B site, respectively. Then the transfer integral between neighboring atoms at $\boldsymbol{R}_{A}$ and $\boldsymbol{R}_{A}-\vec{\tau}_{l}$ becomes

$$
\gamma_{\boldsymbol{R}_{A}, \boldsymbol{R}_{A}-\vec{\tau}_{l}}=\gamma_{0}+\delta \gamma
$$

with

$$
\begin{aligned}
\delta \gamma & =\frac{\partial \gamma_{0}(b)}{\partial b}\left[\left|\vec{\tau}_{l}+\boldsymbol{u}_{A}\left(\boldsymbol{R}_{A}\right)-\boldsymbol{u}_{B}\left(\boldsymbol{R}_{A}-\vec{\tau}_{l}\right)\right|-b\right] \\
& =-\frac{\beta \gamma_{0}}{b^{2}} \vec{\tau}_{l} \cdot\left[\boldsymbol{u}_{A}\left(\boldsymbol{R}_{A}\right)-\boldsymbol{u}_{B}\left(\boldsymbol{R}_{A}-\vec{\tau}_{l}\right)\right]
\end{aligned}
$$

where $b=\left|\vec{\tau}_{l}\right|=a / \sqrt{3}$ and

$$
\beta=-\frac{b}{\gamma_{0}} \frac{\partial \gamma_{0}}{\partial b}=-\frac{d \ln \gamma_{0}}{d \ln b} .
$$

In the following we shall derive an effective-mass equation following the procedure given in ref. 33. First, we should note that for states in the vicinity of the Fermi level $\varepsilon=0$ of the $2 \mathrm{D}$ graphite, the total wavefunction is written as

$$
\begin{gathered}
\psi_{A}\left(\boldsymbol{R}_{A}\right)=\boldsymbol{a}\left(\boldsymbol{R}_{A}\right)^{+} \boldsymbol{F}_{A}\left(\boldsymbol{R}_{A}\right) \\
\psi_{B}\left(\boldsymbol{R}_{B}\right)=\boldsymbol{b}\left(\boldsymbol{R}_{B}\right)^{+} \boldsymbol{F}_{B}\left(\boldsymbol{R}_{B}\right)
\end{gathered}
$$


with

$$
\begin{aligned}
& \boldsymbol{a}\left(\boldsymbol{R}_{A}\right)^{+}=\left(\begin{array}{ll}
e^{i \boldsymbol{K} \cdot \boldsymbol{R}_{A}} & e^{i \eta} e^{i \boldsymbol{K}^{\prime} \cdot \boldsymbol{R}_{A}}
\end{array}\right), \\
& \boldsymbol{b}\left(\boldsymbol{R}_{B}\right)^{+}=\left(\begin{array}{ll}
-\omega e^{i \eta} e^{i \boldsymbol{K} \cdot \boldsymbol{R}_{B}} & e^{i \boldsymbol{K}^{\prime} \cdot \boldsymbol{R}_{B}}
\end{array}\right),
\end{aligned}
$$

and

$$
\boldsymbol{F}_{A}=\left(\begin{array}{c}
F_{A}^{K} \\
F_{A}^{K^{\prime}}
\end{array}\right), \quad \boldsymbol{F}_{B}=\left(\begin{array}{c}
F_{B}^{K} \\
F_{B}^{K^{\prime}}
\end{array}\right),
$$

where $F_{A}^{K}, F_{B}^{K}, F_{A}^{K^{\prime}}$, and $F_{B}^{K^{\prime}}$ are slowly-varying envelope functions.

Introduce a smoothing function $g(\boldsymbol{r})$ which varies smoothly in the range $|r| \lesssim a$ and decays rapidly for $|r| \gg$ a. It should satisfy the conditions:

$$
\sum_{\boldsymbol{R}_{A}} g\left(\boldsymbol{r}-\boldsymbol{R}_{A}\right)=\sum_{\boldsymbol{R}_{B}} g\left(\boldsymbol{r}-\boldsymbol{R}_{B}\right)=1
$$

and

$$
\int d \boldsymbol{r} g\left(\boldsymbol{r}-\boldsymbol{R}_{A}\right)=\int d \boldsymbol{r} g\left(\boldsymbol{r}-\boldsymbol{R}_{B}\right)=\Omega_{0},
$$

where $\Omega_{0}$ is the area of a unit cell given by $\Omega_{0}=\sqrt{3} a^{2} / 2$. The function $g(\boldsymbol{r}-\boldsymbol{R})$ can be replaced by a delta function when it is multiplied by a smooth function such as envelopes, i.e., $g(\boldsymbol{r}-\boldsymbol{R}) \approx \Omega_{0} \delta(\boldsymbol{r}-\boldsymbol{R})$.

We substitute eq. (3.7) into eq. (3.3). Consider the first of such equations. Multiply it by $g\left(\boldsymbol{r}-\boldsymbol{R}_{A}\right) \boldsymbol{a}\left(\boldsymbol{R}_{A}\right)$ and then sum it over $\boldsymbol{R}_{A}$. The term in the left hand side becomes

$$
\sum_{\boldsymbol{R}_{A}} g\left(\boldsymbol{r}-\boldsymbol{R}_{A}\right) \boldsymbol{a}\left(\boldsymbol{R}_{a}\right) \varepsilon \psi_{A}\left(\boldsymbol{R}_{A}\right)=\varepsilon\left(\begin{array}{c}
F_{A}^{K}(\boldsymbol{r}) \\
F_{A}^{K^{\prime}}(\boldsymbol{r})
\end{array}\right) .
$$

The term proportional to $\gamma_{0}$ in the right hand side becomes

$$
\begin{aligned}
& -\gamma_{0} \sum_{\boldsymbol{R}_{A}} g\left(\boldsymbol{r}-\boldsymbol{R}_{A}\right) \boldsymbol{a}\left(\boldsymbol{R}_{a}\right) \sum_{l} \psi_{B}\left(\boldsymbol{R}_{A}-\vec{\tau}_{l}\right) \\
& =\gamma\left(\begin{array}{cc}
e^{i \eta}\left(\hat{k}_{x}-i \hat{k}_{y}\right) & 0 \\
0 & e^{-i \eta}\left(\hat{k}_{x}+i \hat{k}_{y}\right)
\end{array}\right)\left(\begin{array}{c}
F_{B}^{K}(\boldsymbol{r}) \\
F_{B}^{K^{\prime}}(\boldsymbol{r})
\end{array}\right),
\end{aligned}
$$

Finally, the term proportional to $\delta \gamma$ in the right hand side of the first equation of eq. (3.3) is calculated as

$$
\begin{aligned}
\frac{\beta \gamma_{0}}{b^{2}} \sum_{l} & \left(\begin{array}{cc}
-\omega e^{i \eta} e^{-i \boldsymbol{K} \cdot \vec{\tau}_{l}} & 0 \\
0 & e^{-i \eta} e^{-i \boldsymbol{K}^{\prime} \cdot \vec{\tau}_{l}}
\end{array}\right) \\
& \times \vec{\tau}_{l} \cdot\left[\boldsymbol{u}_{A}(\boldsymbol{r})-\boldsymbol{u}_{B}\left(\boldsymbol{r}-\vec{\tau}_{l}\right)\right] \boldsymbol{F}_{B}(\boldsymbol{r}),
\end{aligned}
$$

where $\boldsymbol{u}(\boldsymbol{r})$ is the slowly-varying lattice displacement as a function of the continuous valuable $\boldsymbol{r}$.

Because $\boldsymbol{u}_{A}(\boldsymbol{r})-\boldsymbol{u}_{B}\left(\boldsymbol{r}-\vec{\tau}_{l}\right)$ involves displacements of different sublattices, it has a contribution of optical modes as well as that of acoustic modes and in general $\boldsymbol{u}_{A}(\boldsymbol{r})-\boldsymbol{u}_{B}\left(\boldsymbol{r}-\vec{\tau}_{l}\right) \neq \boldsymbol{u}(\boldsymbol{r})-\boldsymbol{u}\left(\boldsymbol{r}-\vec{\tau}_{l}\right)$. In the long-wavelength limit, however, we can set

$$
\begin{aligned}
& \sum_{l} \exp \left(-i \boldsymbol{K} \cdot \vec{\tau}_{l}\right) \vec{\tau}_{l} \cdot\left[\boldsymbol{u}_{A}(\boldsymbol{r})-\boldsymbol{u}_{B}\left(\boldsymbol{r}-\vec{\tau}_{l}\right)\right] \\
= & \kappa \sum_{l} \exp \left(-i \boldsymbol{K} \cdot \vec{\tau}_{l}\right) \vec{\tau}_{l} \cdot\left[\boldsymbol{u}(\boldsymbol{r})-\boldsymbol{u}\left(\boldsymbol{r}-\vec{\tau}_{l}\right)\right] \\
= & \kappa \sum_{l} \exp \left(-i \boldsymbol{K} \cdot \vec{\tau}_{l}\right)\left(\vec{\tau}_{l} \cdot \vec{\nabla}\right) \vec{\tau}_{l} \cdot \boldsymbol{u}(\boldsymbol{r}),
\end{aligned}
$$

where $\kappa$ is a constant. This $\kappa$ depends on details of a microscopic model of phonons and becomes $\kappa \sim 1 / 3$, smaller than unity, in a valence-force-field model ${ }^{34}$ as is shown in $\S 6$. This reduction factor has been pointed out independently by Woods and Mahan. ${ }^{35}$ We have $\kappa=0$ in a model in which force constants only for change in the distance between carbon atoms are nonzero as shown in $\S 6$.

Now, we shall use the identity

$$
\sum_{l} e^{-i \boldsymbol{K} \cdot \vec{\tau}_{l}}\left(\begin{array}{c}
\left(\tau_{l}^{x}\right)^{2} \\
\tau_{l}^{x} \tau_{l}^{y} \\
\left(\tau_{l}^{y}\right)^{2}
\end{array}\right)=\frac{\omega^{-1}}{4} a^{2}\left(\begin{array}{c}
-1 \\
-i \\
+1
\end{array}\right)
$$

and

$$
\sum_{l} e^{-i \boldsymbol{K}^{\prime} \cdot \vec{\tau}_{l}}\left(\begin{array}{c}
\left(\tau_{l}^{x}\right)^{2} \\
\tau_{l}^{x} \tau_{l}^{y} \\
\left(\tau_{l}^{y}\right)^{2}
\end{array}\right)=\frac{1}{4} a^{2}\left(\begin{array}{c}
-1 \\
+i \\
+1
\end{array}\right)
$$

Then, we have

$$
\frac{3 \kappa \beta}{4} \gamma_{0}\left(\begin{array}{cc}
e^{i \eta}\left(\frac{\partial}{\partial x}+i \frac{\partial}{\partial y}\right) & \left(u_{x}+i u_{y}\right) \\
0 & -e^{-i \eta}\left(\frac{\partial}{\partial x}-i \frac{\partial}{\partial y}\right)\left(u_{x}-i u_{y}\right)
\end{array}\right)
$$

The above quantities are those in the coordinate system fixed onto the graphite sheet and become in the coordinate system defined in the nanotube

$$
\begin{aligned}
& u_{x} \pm i u_{y} \rightarrow e^{ \pm i \eta}\left(u_{x} \pm i u_{y}\right), \\
& \frac{\partial}{\partial x} \pm i \frac{\partial}{\partial y} \rightarrow e^{ \pm i \eta}\left(\frac{\partial}{\partial x} \pm i \frac{\partial}{\partial y}\right) \\
& \hat{k}_{x} \pm i \hat{k}_{y} \rightarrow e^{ \pm i \eta}\left(\hat{k}_{x} \pm i \hat{k}_{y}\right) .
\end{aligned}
$$

Similar expressions can be obtained for $\boldsymbol{F}_{B}$ and the effectivemass equations in the presence of electron-phonon interaction become

$$
\mathcal{H}_{K} \boldsymbol{F}^{K}(\boldsymbol{r})=\varepsilon \boldsymbol{F}^{K}(\boldsymbol{r}), \quad \mathcal{H}_{K^{\prime}} \boldsymbol{F}^{K^{\prime}}(\boldsymbol{r})=\varepsilon \boldsymbol{F}^{K^{\prime}}(\boldsymbol{r}) .
$$

The Hamiltonians become

$$
\begin{aligned}
\mathcal{H}_{K} & =\gamma\left(\begin{array}{cc}
0 & \hat{k}_{x}-i \hat{k}_{y} \\
\hat{k}_{x}+i \hat{k}_{y} & 0
\end{array}\right)+\left(\begin{array}{cc}
V_{1} & V_{2} \\
V_{2}^{*} & V_{1}
\end{array}\right), \\
\mathcal{H}_{K^{\prime}} & =\gamma\left(\begin{array}{cc}
0 & \hat{k}_{x}+i \hat{k}_{y} \\
\hat{k}_{x}-i \hat{k}_{y} & 0
\end{array}\right)+\left(\begin{array}{cc}
V_{1} & -V_{2}^{*} \\
-V_{2} & V_{1}
\end{array}\right),
\end{aligned}
$$

with

where

$$
V_{2}=g_{2} e^{3 i \eta}\left(u_{x x}-u_{y y}+2 i u_{x y}\right),
$$

$$
g_{2}=\frac{3 \kappa \beta}{4} \gamma_{0}
$$

In the above Hamiltonian the deformation potential has also been included.

Usually, we have $\beta \sim 2$ for transfer integral between $p$ orbitals based on the correspondence between a tightbinding model and a nearly-free-electron model with a pseudopotential, ${ }^{34}$ and $\kappa \sim 1 / 3$, which give $g_{2} \sim \gamma_{0} / 2$ or $g_{2} \sim 1.5 \mathrm{eV}$. In ref. $36, \beta$ has been calculated to be 3.6 , which gives $g_{2}=2.7 \mathrm{eV}$. This coupling constant is much 
smaller than the deformation potential constant $g_{1} \sim 30$ $\mathrm{eV}$.

\section{RESISTIVITY}

In CN's, the velocity of acoustic phonons is much smaller than that of electrons and elastic-scattering approximation is applicable. Then, what should be considered is only a backward scattering between a right-going state and a left-going state at the Fermi energy. The wave function of states in the vicinity of the Fermi level in metallic nanotubes is given by

$$
\begin{aligned}
\boldsymbol{F}_{s k}^{K} & \left.=\frac{1}{\sqrt{A L}} \exp (i k y) \mid s, k\right), \\
s, k) & =\frac{1}{\sqrt{2}}\left(\begin{array}{c}
-i s(k /|k|) \\
1
\end{array}\right),
\end{aligned}
$$

where $s=+1$ for the conduction band and -1 for the valence band, and $k>0$ and $k<0$ for the right- and left-going wave, respectively. This wave function corresponds to the state with the vanishing wave vector in the circumference direction and therefore phonon modes with $n=0$ can contribute to the scattering. The matrix element of the backward scattering is given apart from the spatial part by

$$
\left(s,-|k|\left|\left(\begin{array}{cc}
V_{1} & V_{2} \\
V_{2}^{*} & V_{1}
\end{array}\right)\right| s,+|k|\right)=-i \operatorname{Re} V_{2} .
$$

This means that the diagonal deformation-potential term does not contribute to the backward scattering as in the case of impurities ${ }^{17,18}$ and only the real part of the much smaller off-diagonal term contributes to the backward scattering.

We have

$$
\operatorname{Re} V_{2}=g_{2}\left[\left(u_{x x}-u_{y y}\right) \cos 3 \eta-2 u_{x y} \sin 3 \eta\right] .
$$

In armchair nanotubes with $\eta=\pi / 6$, we have

$$
\operatorname{Re} V_{2}=-2 g_{2} u_{x y}
$$

and only shear or twist waves contribute to the scattering. In zigzag nanotubes with $\eta=0$, on the other hand,

$$
\operatorname{Re} V_{2}=g_{2}\left(u_{x x}-u_{y y}\right)
$$

and only stretching and breathing modes contribute to the scattering.

In this study, we calculate resistivity which is defined as the inverse of the Boltzmann conductivity $\sigma=$ $4 e^{2} \gamma \tau_{\eta} / \pi \hbar^{2}$ within the relaxation time approximation, where the factor 4 comes from the electron spin and the degeneracy of $K$ and $K^{\prime}$ points. The relaxation time $\tau_{\eta}$ is given by

$$
\frac{1}{\tau_{\eta}}=\frac{2 A}{\hbar \gamma}\left\langle\left|\frac{1}{A L} \int d x d y\left(\operatorname{Re} V_{2}\right) e^{i 2 k y}\right|^{2}\right\rangle_{T}
$$

where $\langle A[\boldsymbol{u}]\rangle_{T}$ denotes the thermal average of the functional $A[\boldsymbol{u}]$ with regards to the phonon system. Because cross terms $\propto 2 u_{x y}\left(u_{x x}-u_{y y}\right)$ vanish identically for modes $n=0$, we have

$$
\frac{1}{\tau_{\eta}}=\frac{1}{\tau_{A}} \sin ^{2} 3 \eta+\frac{1}{\tau_{Z}} \cos ^{2} 3 \eta
$$

where $\tau_{A}$ and $\tau_{Z}$ are the relaxation time for an armchair and zigzag CN with same $R$. This leads to

$$
\rho_{\eta}(T)=\rho_{A}(T) \sin ^{2} 3 \eta+\rho_{Z}(T) \cos ^{2} 3 \eta,
$$

where $\rho_{A}$ and $\rho_{Z}$ are the resistivity of an armchair and zigzag $\mathrm{CN}$.

In an armchair nanotube the breathing mode does not contribute to the scattering and therefore we can safely employ a high temperature approximation for the phonon distribution function for a whole temperature range and have

$$
\rho_{A}(T)=\frac{h}{e^{2}} \frac{1}{L} \frac{g_{2}^{2} k_{B} T}{2 \gamma^{2} \mu},
$$

where $k_{B}$ is the Boltzmann constant. When a hightemperature approximation is adopted for phonon distribution function, we have the equi-partition law

$$
\begin{aligned}
& \left\langle\left|\frac{1}{A L} \int d x d y\left(2 u_{x y}\right) e^{i 2 k y}\right|^{2}\right\rangle_{T} \\
= & \left\langle\left|\frac{1}{A L} \int d x d y\left(u_{x x}-u_{y y}\right) e^{i 2 k y}\right|^{2}\right\rangle_{T},
\end{aligned}
$$

because $\left(2 u_{x y}\right)^{2}$ and $\left(u_{x x}-u_{y y}\right)^{2}$ are equivalent in the Boltzmann weight if the small potential for curvature change is neglected. Therefore, at temperatures much higher than $T_{B}=\hbar \omega_{B} / k_{B}$ the resistivity of a zigzag nanotube becomes same as that of an armchair nanotube with same $R$ or $L$, i.e.,

$$
\rho_{Z}(T)=\rho_{A}(T)
$$

Therefore, the resistivity of a $\mathrm{CN}$ becomes independent of its chirality at high temperature.

Except in armchair nanotubes, the breathing mode contributes to the scattering and their Bose-Einstein distribution starts to manifest itself at temperatures lower than $T_{B}$. At a low temperature where the breathing mode does not contribute to the scattering and therefore the resistivity of a zigzag nanotube becomes smaller than that of an armchair nanotube with same radius, i.e.,

$$
\rho_{Z}(T)=\rho_{A}(T) \frac{B}{B+\mu}=\rho_{A}(T) \frac{\lambda+\mu}{\lambda+2 \mu} .
$$

Figure 4 shows calculated temperature derivative of the resistivity. Because of the small coupling constant $g_{2}$ the absolute value of the resistivity is much smaller than that in bulk 2D graphite dominated by much larger deformation-potential scattering. The resistivity of an armchair $\mathrm{CN}$ is same as that obtained previously except that $\kappa=1$ has been assumed in the expression of $g_{2} \cdot{ }^{37}$ by

The mean free path $\Lambda$ at high temperature is given

$$
\Lambda=\frac{\mu a^{2}}{3 k_{B} T \kappa^{2} \beta^{2}} L .
$$

Using the parameters in this study, we obtain $\Lambda \sim L \times$ 
$6 \times 10^{2}$ at room temperature. The mean free path is larger than $1 \mu \mathrm{m}$ for thin $(10,10)$ armchair nanotubes and increases in proportion to $L$ with the increase of $L$. This strongly supports the fact that a metallic CN becomes a one-dimensional ballistic conductor even at room temperature.

Doped semiconducting CN's can also show metallic behavior, but they have large resistivity dependent on the Fermi energy. The electron density per unit length $n_{e}$ determines the Fermi wavenumber as

$$
k_{F}=\frac{\pi n_{e}}{4} .
$$

For the reason of particle-hole symmetry, the Fermi energy is assumed to be positive in this paper. The corresponding Fermi velocity is given by

$$
v_{F}=\left|\frac{\partial \varepsilon_{s}(k)}{\hbar \partial k}\right|_{k_{F}}=\frac{\gamma}{\hbar} \frac{3 k_{F} R}{\sqrt{1+\left(3 k_{F} R\right)^{2}}},
$$

as long as the Fermi level lies in the lowest conduction band. In contrast to metallic CN's, the diagonal deformation potential $g_{1}$ causes electron scattering in semiconducting CN's.

Figure 5 shows resistivity of semiconducting CN's for systems with different ratio of $g_{1}$ to $g_{2}$ in units of the resistivity of an armchair $\mathrm{CN}$ which is always metallic. At small $k_{F} R$, resistivity scales as $\left(k_{F} R\right)^{-2}$ as shown in Fig. 5. This originates from the fact that the resistivity is inversely proportional to the square of the Fermi velocity $v_{F}$ (proportional to $k_{F} R$ for small $k_{F} R$ ). As $k_{F} R$ increases, the dispersion of semiconducting CN's approaches that of metallic CN's and so does the wavefunction. Accordingly, the electron scattering due to the deformation potential becomes smaller with $k_{F} R$. However, the result for $g_{1} / g_{2}=10$ shows that the deformation potential dominates resistivity and off-diagonal potential $g_{2}$ can be neglected completely even if electrons are heavily doped up to the bottom of the next subband.

When we consider only a single band around a Fermi point, $k_{F} R$ should be less than 1 for a metallic $\mathrm{CN}$, and $1 / \sqrt{3}$ for a semiconducting $\mathrm{CN}$. For larger $k_{F} R$, it is necessary to solve the Boltzmann transport equation taking scattering between subbands into account. ${ }^{38}$ For CN's with electron-phonon scattering, the increase of the number of conducting modes gives no enhancement of electronic conduction within the Boltzmann transport theory, because the inter-subband scattering becomes increasingly important than the number of conducting modes. This is the case with the impurity scattering as shown in ref. 38.

\section{POSITIVE MAGNETORESISTANCE}

In the presence of a magnetic field $H$ perpendicular to the tube axis as shown in Fig. 1, we can use the gauge

$$
\boldsymbol{A}=\left(0, \frac{L H}{2 \pi} \sin \frac{2 \pi x}{L}\right),
$$

and the effective field for electrons in a $\mathrm{CN}$ is given by the component perpendicular to the surface, i.e.,

$$
H(x)=H \cos \left(\frac{2 \pi x}{L}\right) .
$$

The parameter characterizing its strength is given by

$$
\alpha=\left(\frac{L}{2 \pi l}\right)^{2}
$$

where $l$ is the magnetic length defined by $l=\sqrt{c \hbar / e H}$. In the case $\alpha \ll 1$, the field can be regarded as a small perturbation, while in the case $\alpha \gg 1$, Landau levels are formed on the cylinder surface. Now, plane waves in a circumference direction are not eigenstates and wave functions around the $K$ point are written as

$$
\boldsymbol{F}_{s k}^{K}=\frac{1}{\sqrt{2 A}}\left(\begin{array}{c}
F_{s k}^{K A}(x) \\
F_{s k}^{K B}(x)
\end{array}\right) \exp (i k y) .
$$

where $s=+1$ and -1 for the conduction and valence band, respectively.

The energy levels and wavefunctions are analytically obtained for $\varepsilon=0 .{ }^{39}$

$$
\begin{aligned}
& F_{s k}^{K A}(x)=-i s(k /|k|) F_{-}(x), \\
& F_{s k}^{K B}(x)=F_{+}(x),
\end{aligned}
$$

with

$$
F_{ \pm}(x)=\frac{1}{\sqrt{L I_{0}(2 \alpha)}} \exp \left( \pm \alpha \cos \frac{2 \pi x}{L}\right)
$$

where $I_{0}(z)$ is the modified Bessel function of the first kind defined as

$$
I_{0}(z)=\int_{0}^{\pi} \frac{d \theta}{\pi} \exp (z \cos \theta) .
$$

The corresponding eigenenergies are given by $\varepsilon_{s}(k)=$ $s \gamma|k| / I_{0}(2 \alpha)$, which gives the group velocity $v=\gamma / \hbar I_{0}(2 \alpha)$, and the density of states $D(0)=I_{0}(2 \alpha) / \pi \gamma$.

We should note that

$$
I_{0}(2 \alpha) \approx \begin{cases}1+\alpha^{2}+\cdots & (\alpha \ll 1), \\ e^{2 \alpha} / \sqrt{4 \pi \alpha} & (\alpha \gg 1) .\end{cases}
$$

This means that the group velocity for states at $\varepsilon=0$ decreases and consequently the density of states increases exponentially with the increase of the magnetic field in the high-field regime. In high magnetic fields $(\alpha \gg 1)$, $F_{-}(x)$ is localized around $x= \pm L / 2$, i.e., at the bottom side of the cylinder and $F_{+}(x)$ is localized around the top side $x=0$. The wavefunction for the $K^{\prime}$ point can be obtained in a similar manner.

In the presence of magnetic fields, $V_{1}$ and $\operatorname{Im} V_{2}$ also contribute to the matrix element of electron-phonon scattering and, as a result, to the relaxation time. In this section, we consider high temperature and neglect the potential for curvature change. In this case, the relaxation time and the resistivity become independent of the chirality. The relaxation time is given by

$$
\frac{1}{\tau}=\frac{k_{B} T}{\hbar^{2} v_{F}}\left[\frac{g_{1}^{2}}{B} \int d x\left|F_{s,-k_{F}}^{K A}(x)^{*} F_{s, k_{F}}^{K A}(x)+F_{s,-k_{F}}^{K B}(x)^{*} F_{s, k_{F}}^{K B}(x)\right|^{2}\right.
$$




$$
\left.+2 \frac{g_{2}^{2}}{\mu} \int d x\left(\left|F_{s,-k_{F}}^{K A}(x)^{*} F_{s, k_{F}}^{K B}(x)\right|^{2}+\left|F_{s,-k_{F}}^{K B}(x)^{*} F_{s, k_{F}}^{K A}(x)\right|^{2}\right)\right] .
$$

At $\varepsilon_{F}=0$, after analytical solutions are substituted into the above, we obtain

$$
\rho(H)=\frac{h}{8 e^{2}} \frac{2 k_{B} T}{\gamma^{2} L}\left[\frac{g_{1}^{2}}{B}\left(I_{0}(4 \alpha)-1\right)+2 \frac{g_{2}^{2}}{\mu}\right] .
$$

The rapidly increasing function $I_{0}(4 \alpha)$ with regards to the magnetic field clearly shows that huge magnetoresistance is induced by the diagonal electron-phonon scattering potential $g_{1}$. For the system with nonzero doping $\left(k_{F} R>0\right)$, wavefunctions are numerically solved.

Figure 6 shows resistivity $\rho(H)$ determined by the diagonal potential $g_{1}$. Electrons are not backscattered at all in the absence of a magnetic field but this absence of backscattering disappears in magnetic fields, leading to the huge positive magnetoresistance. This is understood by the analogy with the scattering by the long-range impurity potential. ${ }^{17}$ This magnetoresistance decreases with the increase of the doping.

On the other hand, the resistivity due to the offdiagonal interaction $g_{2}$ is independent of the magnetic field without doping $\left(k_{F} R=0\right)$ as shown in Fig. 7. With the increase of the doping, it starts to exhibit a negative magnetoresistance. The reduction of scattering in magnetic fields for nonzero $k_{F}$ for both $g_{1}$ and $g_{2}$ is caused by the change of the wavefunction at nonzero $k_{F}$ resulting in the decrease of overlap between the initial and final states. ${ }^{39,38}$

In a realistic system, $g_{1}$ is much larger than $g_{2}$ as mentioned in $\S 3$. Figure 8 shows the total magnetoresistance for $g_{1} / g_{2}=10$ in a low magnetic field regime. The large positive magnetoresistance is apparent particularly for $k_{F} R=0$ and decreases gradually with the increase of $k_{F} R$.

This behavior in a low magnetic field is understood from the perturbative expansion in terms of small $\alpha=$ $(R / l)^{2}$. Wavefunctions are obtained up to the second order of $\alpha$ and magnetoresistance is given by

$$
\frac{\rho(H)-\rho_{0}}{\rho_{0}}=2\left[\frac{\mu}{B}\left(\frac{g_{1}}{g_{2}}\right)^{2}-4\left(k_{F} R\right)^{2}\right] \alpha^{2} .
$$

Thus, the magnetoresistance decreases as the doping increases because a small negative magnetoresistance appears for nonzero $k_{F} R$ for the component determined by $g_{2}$.

Within the high-temperature approximation, the magnetoresistance due to the phonon scattering is independent of temperature. However, because the deformation potential is generated by stretching and breathing modes, the magnetoresistance becomes dependent on temperature at temperatures comparable to or lower than $T_{B}$. This will not be discussed further.

The huge positive magnetoresistance predicted here can be observed experimentally in multi-wall CN's. For example, the condition $\alpha=(R / l)^{2}=0.25$ is realized in outer shells of the multi-wall CN with $R=26 \AA$ when $H=25 \mathrm{~T}$. Then, the magnetoresistance reaches about
$\Delta \rho / \rho_{0} \sim 1.0$ at $H=10 \mathrm{~T}$ and $\Delta \rho / \rho_{0} \sim 6.7$ at $H=25$ T. For a $(10,10)$ nanotube with $R=6.79 \AA$, we have $\alpha=$ $(R / l)^{2}=1.7 \times 10^{-2}$ even at $H=25 \mathrm{~T}$ and therefore the magnetoresistance remains almost negligible.

Figure 9 shows magnetoresistance of semiconducting CN's with $\sqrt{3} k_{F} R=0.1,0.5$, and 1.0. Doped semiconducting CN's show positive magnetoresistance which slightly depends on $k_{F} R$. Clearly, it is much smaller than that of metallic CN's given by the thin line in Fig. 9. This is because the deformation potential scatters electrons in semiconducting CN's even without magnetic fields as mentioned in the previous section, and the magnetic field does not cause such drastic change of scattering as in metallic CN's.

\section{MICROSCOPIC PHONON MODELS}

\section{A. Valence-Force-Field Model}

In this section, we derive a continuum model for long-wavelength phonons of a two-dimensional graphite sheet based on a valence-force-field model. For a graphite sheet, in-plane and out-of-plane modes are decoupled. For in-plane modes, two force constants are introduced for restoring forces to bond-stretching and bond-angle change in the simplest approximation. ${ }^{40,35}$

The equations of motion are given by

$$
M \Omega_{0} \omega(\boldsymbol{k})^{2} \boldsymbol{U}(\boldsymbol{k})=H_{p h}(\boldsymbol{k}) \boldsymbol{U}(\boldsymbol{k}),
$$

with

$$
\boldsymbol{U}(\boldsymbol{k})=\left(\begin{array}{c}
u_{A}^{x}(\boldsymbol{k}) \\
u_{A}^{y}(\boldsymbol{k}) \\
u_{B}^{x}(\boldsymbol{k}) \\
u_{B}^{y}(\boldsymbol{k})
\end{array}\right)
$$

Here, the dynamical matrix is given by

$$
H_{\mathrm{ph}}(\boldsymbol{k})=H_{\mathrm{bs}}(\boldsymbol{k})+H_{\mathrm{ba}}(\boldsymbol{k})
$$

with

$$
H_{\mathrm{bs}}(\boldsymbol{k})=K_{1}\left(\begin{array}{cccc}
\frac{3}{2} & 0 & h_{1}(\boldsymbol{k}) & h_{2}(\boldsymbol{k}) \\
0 & \frac{3}{2} & h_{2}(\boldsymbol{k}) & h_{3}(\boldsymbol{k}) \\
h_{1}(\boldsymbol{k})^{*} & h_{2}(\boldsymbol{k})^{*} & \frac{3}{2} & 0 \\
h_{2}(\boldsymbol{k})^{*} & h_{3}(\boldsymbol{k})^{*} & 0 & \frac{3}{2}
\end{array}\right)
$$

and

$H_{\mathrm{ba}}(\boldsymbol{k})=K_{2}\left(\begin{array}{cccc}h_{4}(\boldsymbol{k}) & h_{5}(\boldsymbol{k}) & 6 h_{3}(\boldsymbol{k}) & 2 \sqrt{3} h_{2}(\boldsymbol{k})^{*} \\ h_{5}(\boldsymbol{k})^{*} & h_{6}(\boldsymbol{k}) & 2 \sqrt{3} h_{2}(\boldsymbol{k})^{*} & 6 h_{1}(\boldsymbol{k}) \\ 6 h_{3}(\boldsymbol{k})^{*} & 2 \sqrt{3} h_{2}(\boldsymbol{k}) & h_{4}(\boldsymbol{k}) & h_{5}(\boldsymbol{k}) \\ 2 \sqrt{3} h_{2}(\boldsymbol{k}) & 6 h_{1}(\boldsymbol{k})^{*} & h_{5}(\boldsymbol{k})^{*} & h_{6}(\boldsymbol{k})\end{array}\right)$

where $K_{1}$ and $K_{2}$ are force constants for bond stretching 
and bond-angle change, respectively, and

$$
\begin{aligned}
& h_{1}(\boldsymbol{k})=-\frac{3}{2} e^{i k_{y} a / 2 \sqrt{3}} \cos \frac{k_{x} a}{2}, \\
& h_{2}(\boldsymbol{k})=-i \frac{\sqrt{3}}{2} e^{i k_{y} a / 2 \sqrt{3}} \sin \frac{k_{x} a}{2}, \\
& h_{3}(\boldsymbol{k})=-e^{i k_{y} a / \sqrt{3}}-\frac{1}{2} e^{i k_{y} a / 2 \sqrt{3}} \cos \frac{k_{x} a}{2}, \\
& h_{4}(\boldsymbol{k})=7+\sin ^{2} \frac{k_{x} a}{2}+2 \cos \frac{k_{x} a}{2} \cos \frac{\sqrt{3}}{2} k_{y} a, \\
& h_{5}(\boldsymbol{k})=-\sqrt{3} i e^{\sqrt{3} i k_{y} a / 2} \sin \frac{k_{x} a}{2}+i \frac{\sqrt{3}}{2} \sin k_{x} a, \\
& h_{6}(\boldsymbol{k})=9-3 \sin ^{2} \frac{k_{x} a}{2},
\end{aligned}
$$

We expand this matrix $H_{\mathrm{ph}}(\boldsymbol{k})$ in terms of $k_{x} a$ and $k_{y} a$ as follows

$$
H_{\mathrm{ph}}(\boldsymbol{k})=H^{(0)}+H^{(1)}+H^{(2)}+\cdots,
$$

with

$$
\begin{gathered}
H^{(0)}=\frac{3}{2}\left(K_{1}+6 K_{2}\right)\left(\begin{array}{cccc}
1 & 0 & -1 & 0 \\
0 & 1 & 0 & -1 \\
-1 & 0 & 1 & 0 \\
0 & -1 & 0 & 1
\end{array}\right), \quad(6.7) \\
H^{(1)}=i \frac{\sqrt{3}}{4}\left(K_{1}-6 K_{2}\right) a\left(\begin{array}{cccc}
0 & 0 & -k_{y} & -k_{x} \\
0 & 0 & -k_{x} & k_{y} \\
k_{y} & k_{x} & 0 & 0 \\
k_{x} & -k_{y} & 0 & 0
\end{array}\right),
\end{gathered}
$$

and

$$
\begin{aligned}
H^{(2)} & =K_{1} a^{2}\left(\begin{array}{cccc}
0 & 0 & (1 / 16)\left(3 k_{x}^{2}+k_{y}^{2}\right) & (1 / 8) k_{x} k_{y} \\
0 & 0 & (1 / 8) k_{x} k_{y} & (1 / 16)\left(k_{x}^{2}+3 k_{y}^{2}\right) \\
(1 / 16)\left(3 k_{x}^{2}+k_{y}^{2}\right) & (1 / 8) k_{x} k_{y} & 0 & 0 \\
(1 / 8) k_{x} k_{y} & (1 / 16)\left(k_{x}^{2}+3 k_{y}^{2}\right) & 0 & 0
\end{array}\right) \\
& +K_{2} a^{2}\left(\begin{array}{cccc}
-(3 / 4) k_{y}^{2} & (3 / 4) k_{x} k_{y} & (3 / 8)\left(k_{x}^{2}+3 k_{y}^{2}\right) & -(3 / 4) k_{x} k_{y} \\
(3 / 4) k_{x} k_{y} & -(3 / 4) k_{x}^{2} & -(3 / 4) k_{x} k_{y} & (3 / 8)\left(3 k_{x}^{2}+k_{y}^{2}\right) \\
(3 / 8)\left(k_{x}^{2}+3 k_{y}^{2}\right) & -(3 / 4) k_{x} k_{y} & -(3 / 4) k_{y}^{2} & (3 / 4) k_{x} k_{y} \\
-(3 / 4) k_{x} k_{y} & (3 / 8)\left(3 k_{x}^{2}+k_{y}^{2}\right) & (3 / 4) k_{x} k_{y} & -(3 / 4) k_{x}^{2}
\end{array}\right)
\end{aligned}
$$

We solve the eigenvalue problem with the perturbative expansion in the long wavelength limit. Then, the following variables $\boldsymbol{u}(\boldsymbol{r})=\left(u_{x}, u_{y}\right)$ and $\boldsymbol{v}(\boldsymbol{r})=\left(v_{x}, v_{y}\right)$ enable to treat acoustic and optical phonons separately,

$$
\begin{aligned}
& u_{x}=\frac{1}{\sqrt{2}}\left(u_{A}^{x}+u_{B}^{x}\right)-\kappa^{\prime} a \frac{1}{\sqrt{2}}\left[\frac{\partial}{\partial y}\left(u_{A}^{x}-u_{B}^{x}\right)+\frac{\partial}{\partial x}\left(u_{A}^{y}-u_{B}^{y}\right)\right]+\cdots, \\
& u_{y}=\frac{1}{\sqrt{2}}\left(u_{A}^{y}+u_{B}^{y}\right)-\kappa^{\prime} a \frac{1}{\sqrt{2}}\left[\frac{\partial}{\partial x}\left(u_{A}^{x}-u_{B}^{x}\right)-\frac{\partial}{\partial y}\left(u_{A}^{y}-u_{B}^{y}\right)\right]+\cdots, \\
& v_{x}=\frac{1}{\sqrt{2}}\left(u_{A}^{x}-u_{B}^{x}\right)-\kappa^{\prime} a \frac{1}{\sqrt{2}}\left[\frac{\partial}{\partial y}\left(u_{A}^{x}+u_{B}^{x}\right)+\frac{\partial}{\partial x}\left(u_{A}^{y}+u_{B}^{y}\right)\right]+\cdots, \\
& v_{y}=\frac{1}{\sqrt{2}}\left(u_{A}^{y}-u_{B}^{y}\right)-\kappa^{\prime} a \frac{1}{\sqrt{2}}\left[\frac{\partial}{\partial x}\left(u_{A}^{x}+u_{B}^{x}\right)-\frac{\partial}{\partial y}\left(u_{A}^{y}+u_{B}^{y}\right)\right]+\cdots,
\end{aligned}
$$

with

$$
\kappa^{\prime}=\frac{K_{1}-6 K_{2}}{4 \sqrt{3}\left(K_{1}+6 K_{2}\right)}
$$

This linear transformation gives two sets of eigen-equations up to the second order of wavenumbers,

$$
\begin{aligned}
& M \Omega_{0} \omega(\boldsymbol{k})^{2} \boldsymbol{u}(\boldsymbol{k})=H_{\mathrm{ac}}(\boldsymbol{k}) \boldsymbol{u}(\boldsymbol{k}), \\
& M \Omega_{0} \omega(\boldsymbol{k})^{2} \boldsymbol{v}(\boldsymbol{k})=H_{\mathrm{op}}(\boldsymbol{k}) \boldsymbol{v}(\boldsymbol{k}),
\end{aligned}
$$

with

$$
H_{\mathrm{ac}}=\frac{1}{8} K_{1} a^{2}\left(\begin{array}{cc}
k_{x}^{2} & k_{x} k_{y} \\
k_{y} k_{x} & k_{y}^{2}
\end{array}\right)+\frac{3}{2} \frac{K_{1} K_{2}}{K_{1}+6 K_{2}} a^{2}\left(\begin{array}{cc}
k_{x}^{2}+k_{y}^{2} & 0 \\
0 & k_{x}^{2}+k_{y}^{2}
\end{array}\right),
$$

and

$$
\begin{aligned}
H_{\mathrm{op}} & =3\left(K_{1}+6 K_{2}\right)\left(\begin{array}{cc}
1 & 0 \\
0 & 1
\end{array}\right)+\left(-\frac{1}{8} K_{1}+\frac{3}{2} K_{2}\right) a^{2}\left(\begin{array}{cc}
k_{x}^{2} & k_{x} k_{y} \\
k_{y} k_{x} & k_{y}^{2}
\end{array}\right) \\
& -\frac{3}{2} \frac{K_{1}+3 K_{2}}{K_{1}+6 K_{2}} K_{2} a^{2}\left(\begin{array}{cc}
k_{x}^{2}+k_{y}^{2} & 0 \\
0 & k_{x}^{2}+k_{y}^{2}
\end{array}\right) .
\end{aligned}
$$

In a real-space representation, we obtain the potential-energy functional for $\boldsymbol{u}(\boldsymbol{r})$ which is given by eq. (2.1) with strains defined as eq. (2.2). Lamé constants are defined as

$$
\begin{aligned}
\lambda & =\frac{1}{4 \sqrt{3}} \frac{K_{1}-6 K_{2}}{K_{1}+6 K_{2}} K_{1}, \\
\mu & =\sqrt{3} \frac{K_{1} K_{2}}{K_{1}+6 K_{2}} .
\end{aligned}
$$




\section{B. Electron-Phonon Interaction}

As shown in $\S 3$, there appears $\boldsymbol{u}_{A}(\boldsymbol{r})-\boldsymbol{u}_{B}\left(\boldsymbol{r}-\vec{\tau}_{l}\right)$ in electron-phonon interaction. According to the valenceforce-field model in the previous subsection, the variable $\boldsymbol{u}(\boldsymbol{r})$ describing the effective displacement of acoustic modes includes not only the center-of-mass motion between atoms at different sublattices but also their relative displacement. Solving eq. (6.10) inversely, we obtain

$$
\begin{aligned}
& u_{A}^{x}=\frac{1}{\sqrt{2}}\left(u_{x}+v_{x}\right)+\kappa^{\prime} a \frac{1}{\sqrt{2}}\left[\frac{\partial}{\partial y}\left(u_{x}+v_{x}\right)+\frac{\partial}{\partial x}\left(u_{y}+v_{y}\right)\right]+\cdots, \\
& u_{A}^{y}=\frac{1}{\sqrt{2}}\left(u_{y}+v_{y}\right)+\kappa^{\prime} a \frac{1}{\sqrt{2}}\left[\frac{\partial}{\partial x}\left(u_{x}+v_{x}\right)-\frac{\partial}{\partial y}\left(u_{y}+v_{y}\right)\right]+\cdots \\
& u_{B}^{x}=\frac{1}{\sqrt{2}}\left(u_{x}-v_{x}\right)-\kappa^{\prime} a \frac{1}{\sqrt{2}}\left[\frac{\partial}{\partial y}\left(u_{x}-v_{x}\right)+\frac{\partial}{\partial x}\left(u_{y}-v_{y}\right)\right]+\cdots \\
& u_{B}^{y}=\frac{1}{\sqrt{2}}\left(u_{y}-v_{y}\right)-\kappa^{\prime} a \frac{1}{\sqrt{2}}\left[\frac{\partial}{\partial x}\left(u_{x}-v_{x}\right)-\frac{\partial}{\partial y}\left(u_{y}-v_{y}\right)\right]+\cdots
\end{aligned}
$$

and, retaining only contributions from acoustic modes,

$$
\begin{aligned}
& u_{A}^{x}(\boldsymbol{r})-u_{B}^{x}\left(\boldsymbol{r}-\vec{\tau}_{l}\right)=\frac{1}{\sqrt{2}}\left(\vec{\tau}_{l} \cdot \vec{\nabla}\right) u_{x}+\sqrt{2} \kappa^{\prime} a\left(\frac{\partial u_{x}}{\partial y}+\frac{\partial u_{y}}{\partial x}\right)+\cdots \\
& u_{A}^{y}(\boldsymbol{r})-u_{B}^{y}\left(\boldsymbol{r}-\vec{\tau}_{l}\right)=\frac{1}{\sqrt{2}}\left(\vec{\tau}_{l} \cdot \vec{\nabla}\right) u_{y}+\sqrt{2} \kappa^{\prime} a\left(\frac{\partial u_{x}}{\partial x}-\frac{\partial u_{y}}{\partial y}\right)+\cdots
\end{aligned}
$$

We have $\kappa=1 / \sqrt{2}$ when only the first terms in the right hand side are taken into consideration. The second terms make the following contribution to the electron-phonon interaction around the $K$ point

$$
\begin{aligned}
& -\frac{\beta \gamma_{0}}{b^{2}} \omega e^{i \eta} \sqrt{2} \kappa^{\prime} a \sum_{l} e^{-i \boldsymbol{K} \cdot \vec{\tau}_{l}}\left[2 \tau_{l}^{x} u_{x y}+\tau_{l}^{y}\left(u_{x x}-u_{y y}\right)\right] \\
& =-3 \sqrt{\frac{3}{2}} \beta \gamma_{0} e^{i \eta} \kappa^{\prime}\left[\left(u_{x x}-u_{y y}\right)+i 2 u_{x y}\right]
\end{aligned}
$$

which gives nothing but a correction to $\kappa$. Therefore, eq. (3.15) is justified and $\kappa$ is given by

$$
\kappa=\frac{1}{\sqrt{2}}-4 \sqrt{\frac{3}{2}} \kappa^{\prime}=\frac{1}{\sqrt{2}} \frac{12 K_{2}}{K_{1}+6 K_{2}} .
$$

Note that this is proportional to the force constant for bond-angle change and therefore electron-phonon interaction vanishes without restoring force to bond-angle change. This is the case with models containing more force constants as long as restoring force between atom pairs for radial displacement are considered and force constants involving three or more atoms are completely neglected.

\section{Parameter Estimation}

In our model, there exist two parameters for in-plane modes of acoustic phonons. What is necessary for our continuum elastic model are two elastic constants $B$ and $\mu$, and it is of no importance which microscopic model is originally adopted unless optical phonons are concerned. Such parameters of nanotubes have not yet been settled by existing studies. They can also be determined by the velocity of twisting and stretching modes. However, the sound velocity of such modes has not not been obtained experimentally and has not uniquely been fixed theoretically, either. Therefore, it seems to be a good idea to use the data of graphites because our elastic model for nanotubes is based on that for graphite sheets.

By neglecting inter-layer interactions, we adopt the following parameters for bulk graphites: ${ }^{41}$

$$
\begin{aligned}
& B / M=2.90 \times 10^{8} \mathrm{~m}^{2} / \mathrm{s}^{2}, \\
& \mu / M=1.51 \times 10^{8} \mathrm{~m}^{2} / \mathrm{s}^{2}, \\
& \Xi / M=6.19 \times 10^{6} \mathrm{~m}^{2} / \mathrm{s}^{2} .
\end{aligned}
$$

Within this model the parameter $\kappa$ is also given by the above elastic constants,

$$
\kappa=\frac{1}{\sqrt{2}} \frac{\mu}{B}=0.369 .
$$

As has been mentioned in the previous subsection, this $\kappa$ cannot be determined by the elastic theory alone but depends on the original valence-force-field model. Nonetheless, eq. (6.19) is approximately valid for models containing higher-order interactions because there is no correction from the bond-stretching and bond-angle change for the second-nearest-neighbor atoms at the same sublattice. In fact, we have $K_{2} / K_{1}=0.0565$ and, as a result, $\kappa=0.358$ in the model of ref. 42 in which more force constants were introduced than the present two-parameter valence-force-field model. Therefore, our choice $\kappa \sim 1 / 3$ is likely to be quite reasonable.

An extensive study for phonons of a single graphite sheet has been given by ref. 43 and the same model with small corrections due to the curvature has been used to calculate the phonon dispersion of nanotubes. ${ }^{31}$ However, the obtained sound velocities are not compatible with the elastic model because they give a negative Poisson 
ratio which is highly improbable.

\section{DISCUSSION}

There exist several theoretical studies on the dispersion relation of phonons in a $\mathrm{CN}$ with small radius though its experimental observation is still difficult. Our continuum effective model for acoustic phonons demonstrates the breathing mode with the energy proportional to $R^{-1}$ and the existence of four zero modes which cannot be reproduced by the zone-folding method to phonon$\mathrm{s}$ of a graphite sheet. There is a $R^{-2}$ correction to the breathing mode from the potential term due to curvature change, but it is very small even for the $(10,10)$ armchair $\mathrm{CN}$ and negligible for CN's with large radius. These results agree with those of the pioneering study in ref. 31 . The energy of the lowest Raman-active mode has been predicted in ref. 31 to be almost proportional to $R^{-2}$. This corresponds to the modes at $q R=0$ and $n= \pm 2$ with frequency $\omega / \omega_{B} \approx 0.12$ for the $(10,10)$ armchair C$\mathrm{N}$ in Fig. 4(b). This nonzero frequency originates from the potential for curvature change and therefore scales as $R^{-2}$, and the absolute value of the frequency also shows a good agreement.

As mentioned in $\S 2$, the dispersion of the bending modes is proportional to $q^{2}$ in contrast to the result of ref. 31. This $q^{2}$ dispersion is consistent with the elastic theory for bending of cylinders, ${ }^{29}$ more recent results of ab initio calculations, ${ }^{44}$ and results of a lattice-dynamical model. ${ }^{45}$

There are only a few experimental studies reporting normal metallic behavior of temperature dependence of CN's. ${ }^{46}$ With the present experimental technique it seems quite difficult to measure the resistivity of one metallic single-walled $\mathrm{CN}$ for which the chirality is identified. The resistivity of samples containing various CN's shows the temperature dependence of metallic nanotubes reflecting the distribution of chirality as long as Ohmic contact is realized because semiconducting nanotubes with large resistivity make little contributions to electron transport in such samples.

It should be noted that two-dimensional lattice is thermodynamically unstable. ${ }^{47}$ For example, $\left\langle u_{x}^{2}\right\rangle_{T}$ is divergent though $\left\langle\left(\partial u_{x} / \partial x\right)^{2}\right\rangle_{T}$ has a finite value. Therefore, we should consider some environments with threedimensionality or a finite tube length to cut off such a divergence. In fact, CN's usually form bundles, mats, and so on. A single $\mathrm{CN}$ used in experiments always has a finite length and is put on a certain substrate material or attached to a metallic contact. These effects may drastically change phonon modes with small wavenumber. It is possible that electron-phonon scattering in a $\mathrm{CN}$ with $\varepsilon_{F} \sim 0$ shows singular behavior. Clarification of such effects is left for a future study.

\section{SUMMARY AND CONCLUSION}

We have studied electron-phonon scattering in metallic CN's. A continuum elastic model with only three constants has been proposed for long-wavelength acoustic modes playing a major role in electron-phonon scattering. Electron-phonon interaction has been derived based on the effective-mass theory for conducting electrons. The conventional diagonal deformation potential does not contribute to backward scattering and therefore gives rise to no resistance in metallic nanotubes in the absence of a magnetic field. Therefore, we have to consider a small and chirality-dependent off-diagonal potential due to local modification of band structure.

In metallic CN's, only the off-diagonal potential scatters electrons in the absence of a magnetic field. For armchair CN's, only a twisting mode causes scattering. On the other hand, stretching and breathing modes contribute to scattering for zigzag CN's, At high temperature, contribution from both modes in a zigzag $\mathrm{CN}$ is equal to that from a twisting mode in an armchair CN. The breathing mode with finite energy gap is hardly excited and causes little scattering at temperatures lower than its energy. As a result the resistivity of a metallic $\mathrm{CN}$ is dependent on the chirality.

In a magnetic field electron scattering is induced even in metallic CN's by the diagonal deformation potential which is much larger than the off-diagonal potential, leading to a huge positive magnetoresistance. This can be observed easily in a metallic $\mathrm{CN}$ with large radius.

\section{ACKNOWLEDGMENTS}

This work has been supported in part by Grantsin-Aid for COE (12CE2004 "Control of Electrons by Quantum Dot Structures and Its Application to Advanced Electronics"), Scientific Research, and Priority Area (Fullerene Network) from the Ministry of Education, Science and Culture, Japan.

\section{References}

1 S. Iijima, Nature (London) 354, 56 (1991).

2 N. Hamada, S. Sawada, and A. Oshiyama, Phys. Rev. Lett. 68, 1579 (1992).

3 J. W. Mintmire, B. I. Dunlap, and C. T. White, Phys. Rev. Lett. 68, 631 (1992).

4 R. Saito, M. Fujita, G. Dresselhaus, and M. S. Dresselhaus, Phys. Rev. B 46, 1804 (1992); Appl. Phys. Lett. 60, 2204 (1992).

5 M. S. Dresselhaus, G. Dresselhaus, and R. Saito, Phys. Rev. B 45, 6234 (1992).

6 R. A. Jishi, M. S. Dresselhaus, and G. Dresselhaus, Phys. Rev. B 47, 16671 (1993).

7 K. Tanaka, K. Okahara, M. Okada and T. Yamabe: Chem. Phys. Lett. 191, 469 (1992).

8 Y. D. Gao and W. C. Herndon: Mol. Phys. 77, 585 (1992).

9 D. H. Robertson, D. W. Brenner, and J. W. Mintmire, Phys. Rev. B 45, 12592 (1992).

10 C. T. White, D. C. Robertson, and J. W. Mintmire, Phys. Rev. B 47, 5485 (1993).

11 H. Ajiki and T. Ando, J. Phys. Soc. Jpn. 62, 1255 (1993).

12 A. M. Rao, E. Richter, S. Bandow, B. Chase, P. C. Eklund, K. W. Williams, M. Menon, K. R. Subbaswamy, A. Thess, R. E. Smalley, G. Dresselhaus, and M. S. Dresselhaus, Science 275, 187 (1997).

13 C. H. Olk and J. P. Heremans, J. Mater. Res. 9, 259 (1994).

14 J. W. Wildoer, L. C. Venema, A. G. Rinzler, R. E. Smalley, and C. Dekker, Nature (London) 391, 59 (1998).

15 A. Hassanien, M. Tokumoto, Y. Kumazawa, H. Kataura, Y. Maniwa, S. Suzuki, and Y. Achiba, Appl. 
Phys. Lett. 73, 3839 (1998).

16 T. Ando, Semicond. Sci. Technol. 15, R13 (2000).

17 T. Ando and T. Nakanishi, J. Phys. Soc. Jpn. 67, 1704 (1998)

18 Y. Ando, X. -L. Zhao, and M. Ohkohchi, Jpn. J. Appl. Phys. 37, L61 (1998).

19 T. Nakanishi and T. Ando, J. Phys. Soc. Jpn. 68, 561 (1999).

20 P. L. McEuen, M. Bockrath, D. H. Cobden, Y. -G. Yoon, and S. G. Louie, Phys. Rev. Lett. 83, 5098 (1999).

21 A. Bachtold, M. S. Fuhrer, S. Plyasunov, M. Forero, E. H. Anderson, A. Zettl, and P. L. McEuen, Phys. Rev. Lett. 84, 6082 (2000).

22 L. Chico, L. X. Benedict, S. G. Louie, and M. L. Cohen, Phys. Rev. B 54, 2600 (1996).

23 M. Igami, T. Nakanishi, and T. Ando, J. Phys. Soc. Jpn. 68, 716 (1999).

24 M. Igami, T. Nakanishi, and T. Ando, J. Phys. Soc. Jpn. 68, 3146 (1999).

25 T. Ando, T. Nakanishi, and M. Igami, J. Phys. Soc. Jpn. 68, 3994 (1999).

26 M. Igami, T. Nakanishi, and T. Ando, J. Phys. Soc. Jpn. 70, 481 (2001).

27 H. Suzuura and T. Ando, Physica E 6, 864 (2000).

28 H. Suzuura and T. Ando, Mol. Cryst. and Liq. Cryst. 340, 731 (2000).

29 L. D. Landau and E. M. Lifshitz, Theory of elasticity (Pergamon Press, New York, 1970).

30 P. M. Chaikin and T. C. Lubensky, Principles of condensed matter physics (Cambridge University Press, Cambridge, 1995).

31 R. Saito, T. Takeya, and T. Kimura, G. Dresselhaus, and M. S. Dresselhaus, Phys. Rev. B 57, 4145 (1998).

32 K. Sugihara, Phys. Rev. B 28, 2157 (1983).

33 H. Matsumura and T. Ando, J. Phys. Soc. Jpn. 70, 2401 (2001).

34 See for example, W. A. Harrison, Electronic Structure and the Properties of Solids (W.H. Freeman and Company, San Francisco, 1980).

35 L. M. Woods and G. D. Mahan, Phys. Rev. B 61, 10651 (2000).

36 L. Pietronero, S. Strässler, and H. R. Zeller, Phys. Rev. B 22, 904 (1980).

37 C. L. Kane, E. J. Mele, R. S. Lee, J. E. Fischer, P. Petit, H. Dai, A. Thess, R. E. Smalley, A. R. M. Verschueren, S. J. Tans, and C. Dekker, Europhys. Lett. 41, 683 (1998).

38 T. Seri and T. Ando, J. Phys. Soc. Jpn. 66, 169 (1997).

39 T. Ando and T. Seri, J. Phys. Soc. Jpn. 66, 3558 (1997).

40 A. Yoshimori and Y. Kitano, J. Phys. Soc. Jpn. 11, 352 (1956)

41 K. Komatsu, J. Phys. Chem. Solids 25, 707 (1964).

42 T. Aizawa, R. Souda, S. Otani, and Y. Ishizawa, and C. Oshima, Phys. Rev. B 42, 11469 (1990).

43 R. A. Jishi, L. Venkataraman, M. S. Dresselhaus, and G. Dreselhaus, Chem. Phys. Lett. 209, 77 (1993)

44 D. Sanchez-Portal, E. Artacho, and J. M. Soler,
A. Rubio, and P. Ordejo, Phys. Rev. B 59, 12678 (1999).

45 V. N. Popov, V. E. Van Doren, and M. Balkanski, Phys. Rev. B 61, 3078 (2000).

46 J. E. Fischer, H. Dai, A. Thess, R. Lee, N. M. Hanjani, D. L. Dehaas, and R. E. Smalley, Phys. Rev. B 55, R4921 (1997).

47 L. D. Landau and E. M. Lifshitz, Statistical Physics (Pergamon Press, New York, 1980).

\section{Figure Captions}

Fig. 1. The lattice structure of a $2 \mathrm{D}$ graphite. A unit cell contains two carbon atoms denoted as $\mathrm{A}$ and $\mathrm{B}$ and two primitive translation vectors are denoted as $\boldsymbol{a}$ and $\boldsymbol{b}$. The coordinate system $(x, y)$ is chosen in such a way that the $y$ axis is along the tube axis and the $x$ axis is along the circumference. The chiral vector corresponding to a circumference of the tube is denoted as $\boldsymbol{L}$ and the chiral angle $\eta$ is that between the chiral vector and the $x$ axis fixed on the $2 \mathrm{D}$ graphite.

Fig. 2. Some examples of deformation of the cross section of CN with $n=0, \pm 1$, and \pm 2 .

Fig. 3. Frequencies of phonons for the $(10,10)$ armchair $\mathrm{CN}$ obtained in the continuum model. (a) Without out-of-plane curvature effect and (b) with out-ofplane curvature effect.

Fig. 4. Temperature derivative of resistivity of armchair (solid line), chiral (dotted line), and zigzag (broken line) nanotubes in units of $d \rho_{A}\left(T_{B}\right) / d T$ which is the temperature derivative of resistivity of the armchair nanotube at $T=T_{B}$, and $T_{B}$ denotes the temperature of the breathing mode, $T_{B}=\hbar \omega_{B} / k_{B}$.

Fig. 5. Electron-density dependence of resistivity for semiconducting CN's with $g_{1} / g_{2}=10$ (solid line), 5 (dotted line), and 1 (broken line) in units of $\rho_{A}$ denoting the resistivity of an armchair $\mathrm{CN}$ which is independent of electron density (thin solid line).

Fig. 6. Resistivity with a fixed electron density in magnetic fields determined by the diagonal deformation potential. The unit $\rho_{g 1}$ is defined so that $\rho(H)$ becomes $\rho_{g 1}\left[I_{0}(4 \alpha)-1\right]$ for $k_{F} R=0$ as given in eq. (5.10).

Fig. 7. Resistivity with a fixed electron density in magnetic fields determined by the off-diagonal term. The unit $\rho_{0}$ is the resistivity at $H=0$.

Fig. 8. Calculated magnetoresistance in a low-magneticfield region.

Fig. 9. Magnetoresistance of semiconducting CN's with $\sqrt{3} k_{F} R=0.1$ (solid line), 0.5 (dotted line), and 1.0 (broken line). The thin solid line shows magnetoresistance of an armchair CN with $\sqrt{3} k_{F} R=1$. 

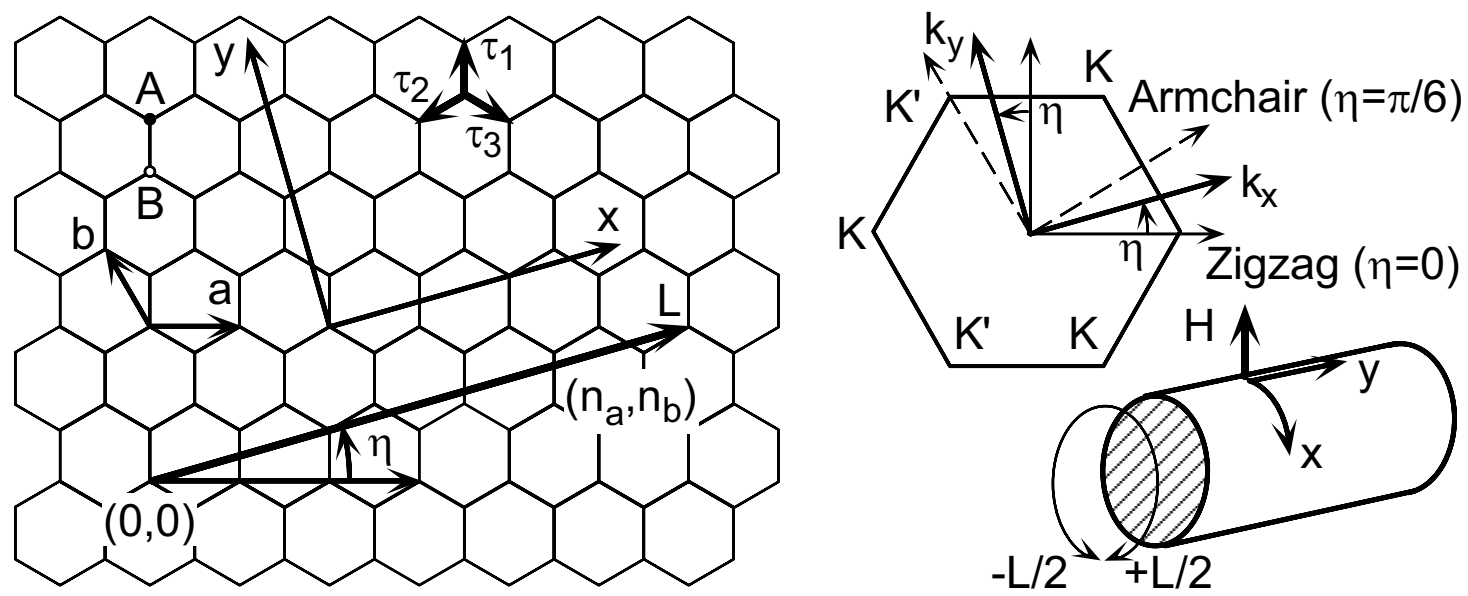

Fig. 1
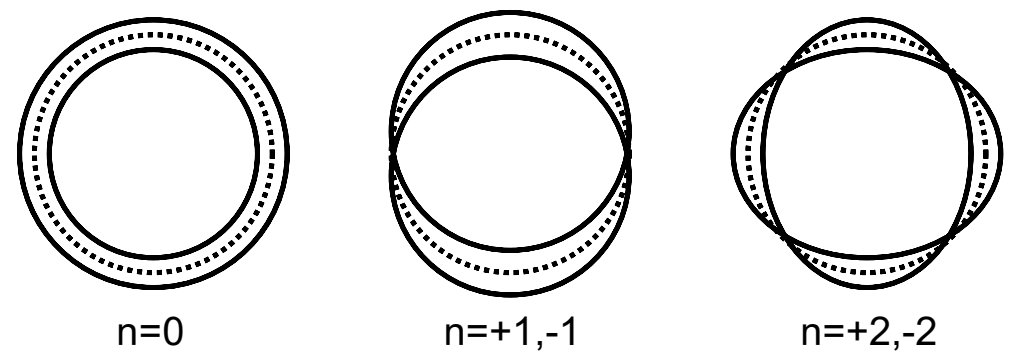

Fig. 2

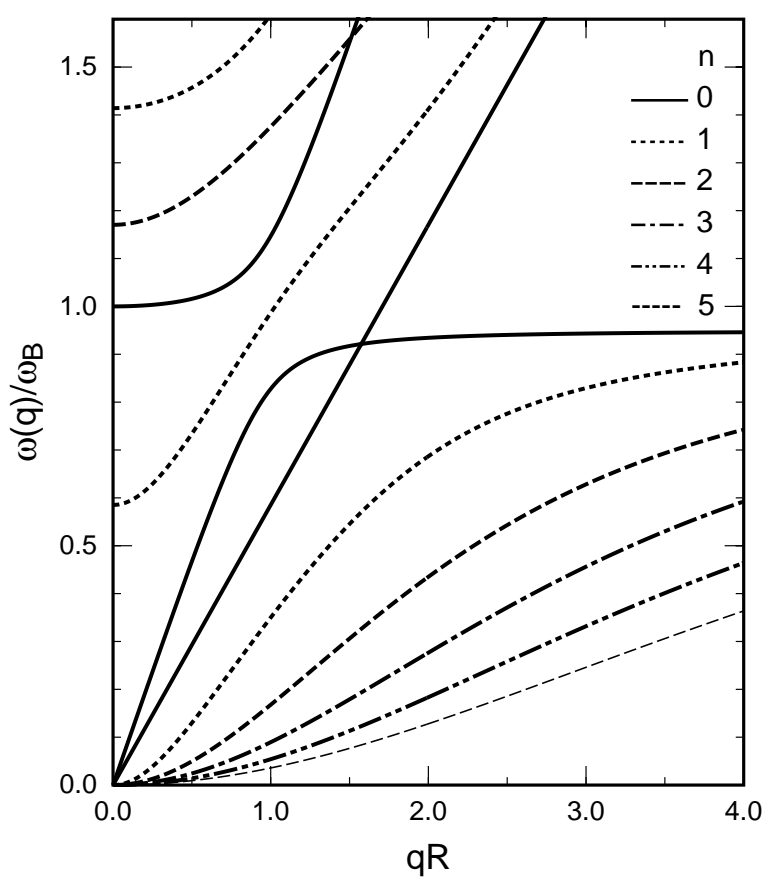

Fig. 3(a)

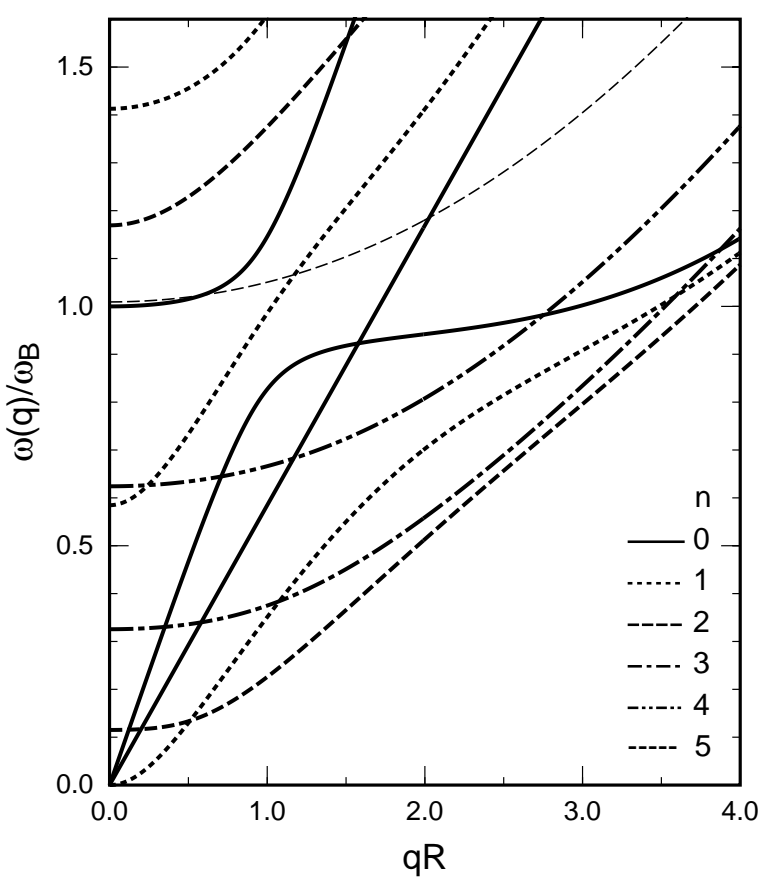

Fig. 3(b) 


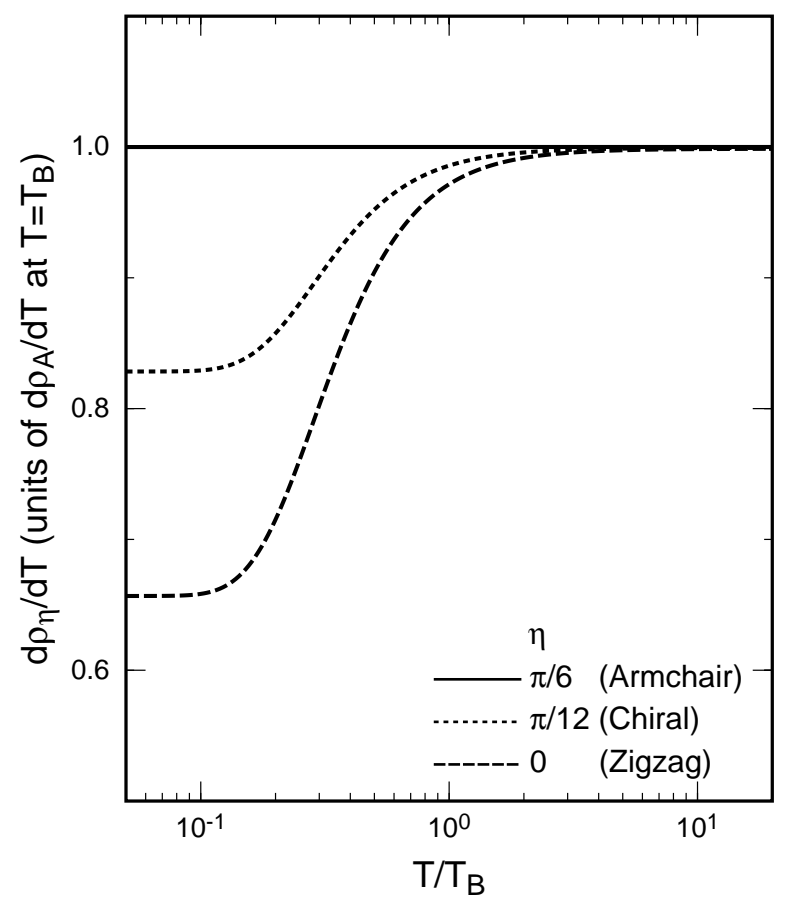

Fig. 4

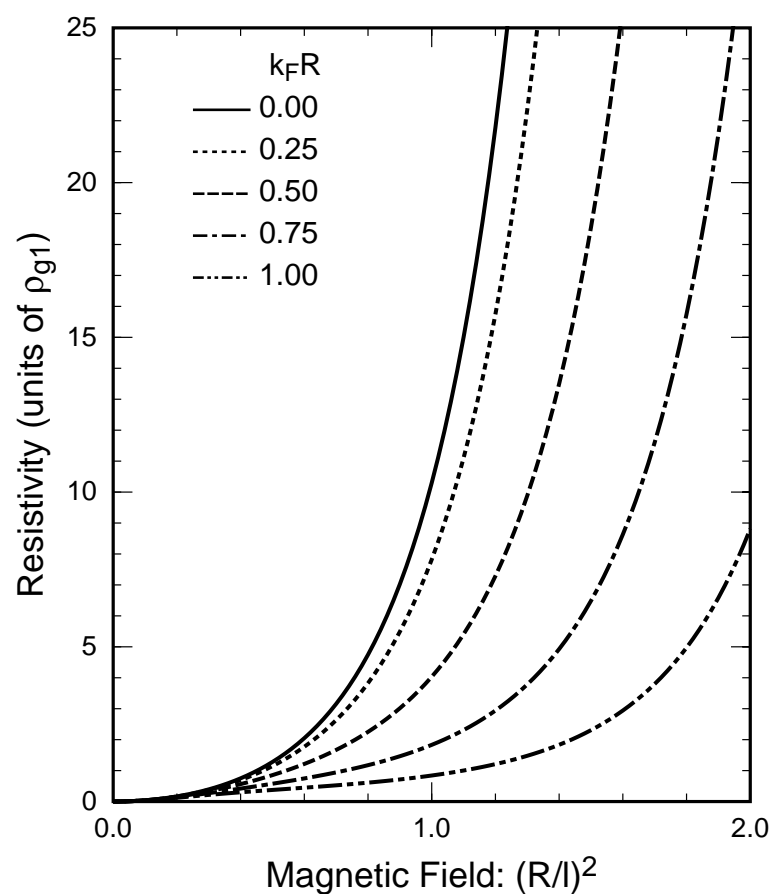

Fig. 6

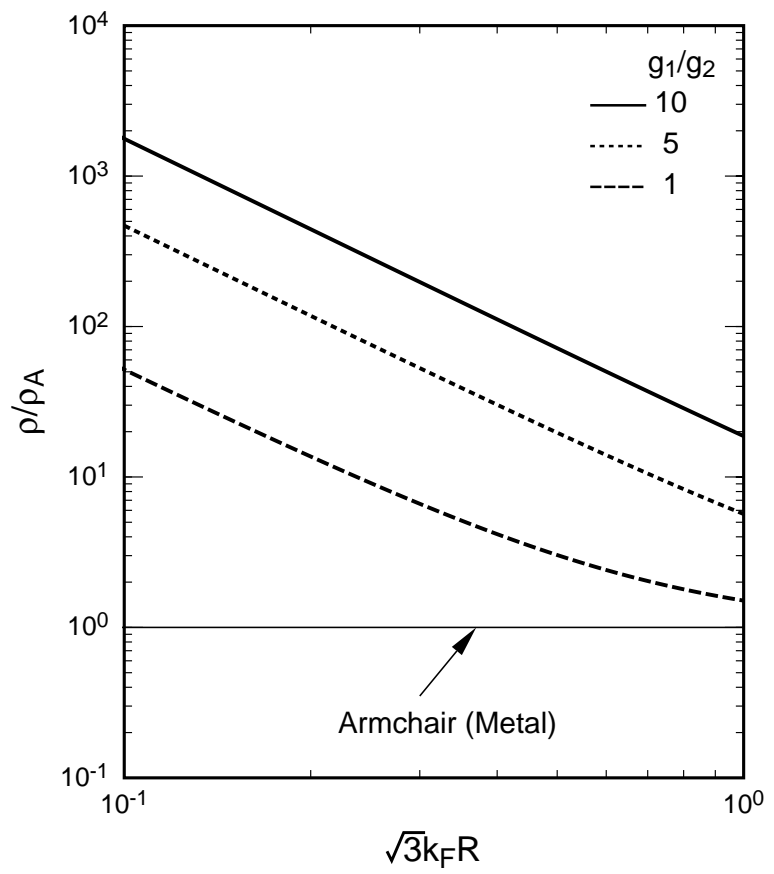

Fig. 5

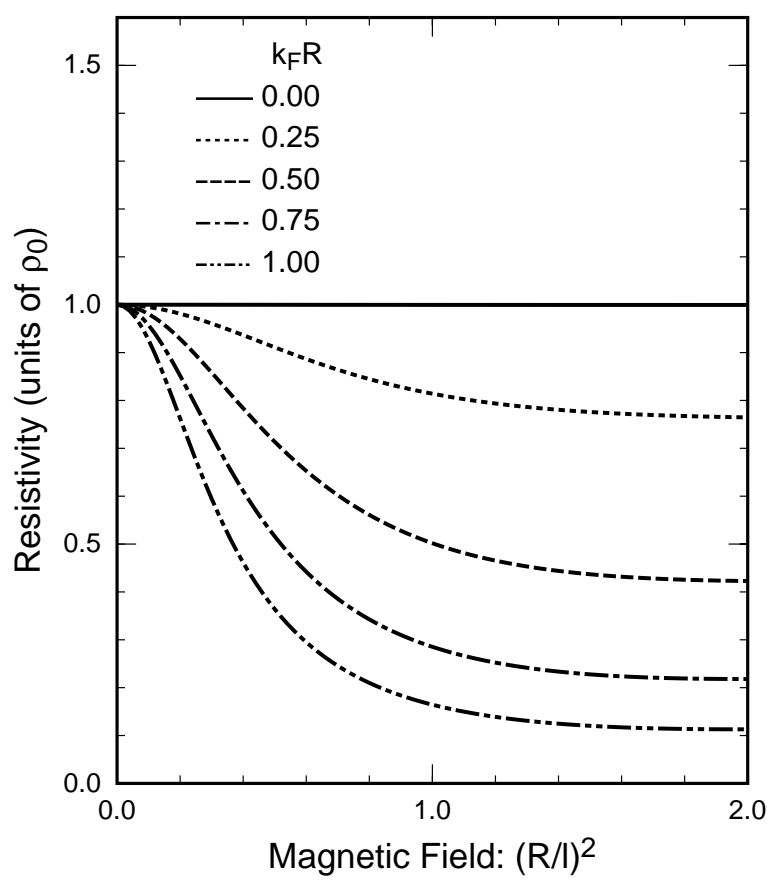

Fig. 7 


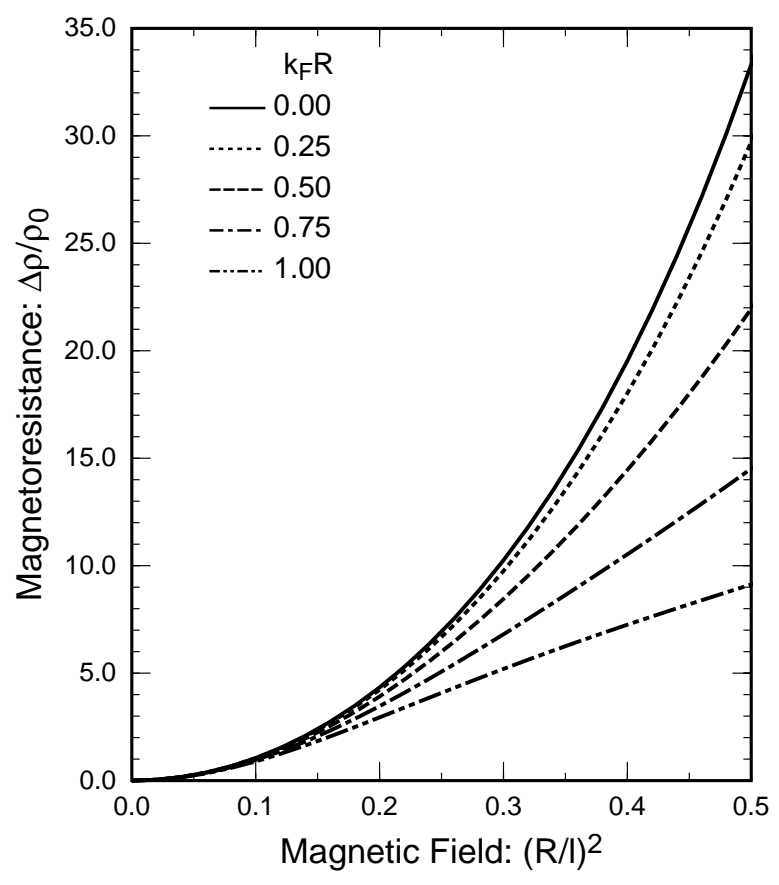

Fig. 8

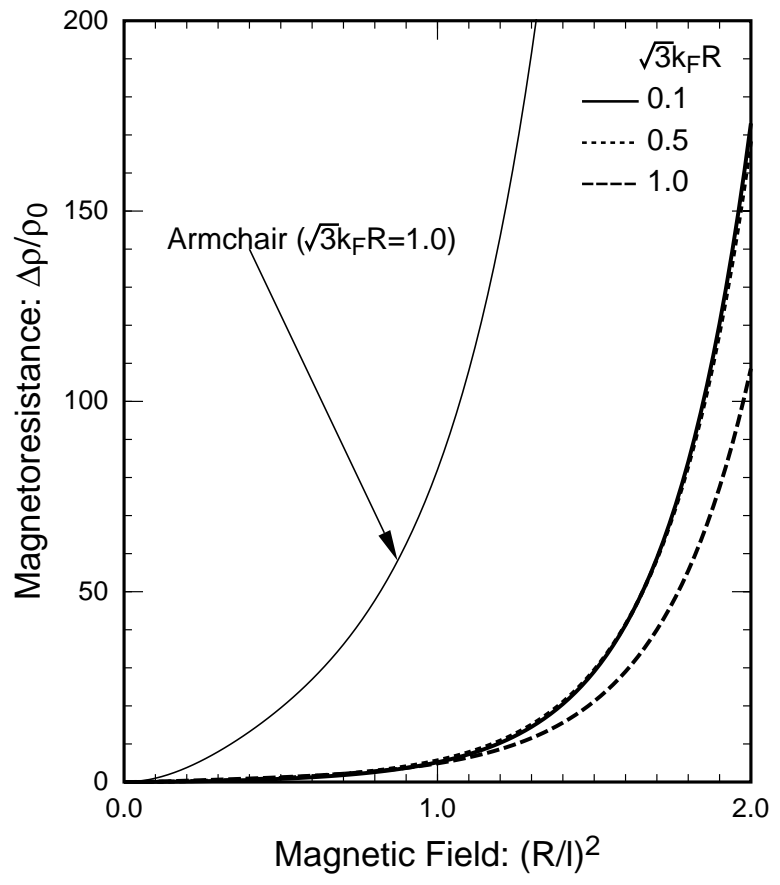

Fig. 9 